\title{
Comparision of ZN Staining and Fluorescent Microscopy in Detection of Acid Fast Bacilli in Fine Needle Aspiration Smears
}

\author{
Vikas Dagar $^{1}$, Shruti Heda ${ }^{1}$, Ashwinkumar Barsagade ${ }^{1}$, Sadhana Mahore $^{2}$, \\ Nitin Ambhore ${ }^{3}$, Rajesh Karyakarte ${ }^{4}$, Akansha Salkar ${ }^{1}$, \\ Kalpana Bothale ${ }^{1}$, Sameer Khan ${ }^{1}$ \\ ${ }^{1}$ (Assistant Professor, Pathology Department, GMC Akola, India) \\ ${ }^{2}$ (Professor And Head, Microbiology Department, NKP Salve Nagpur, India) \\ ${ }_{3}^{3}$ (Associate Professor, Microbiology Department, GMC Akola, India) \\ ${ }^{4}$ (Professor, Microbiology Department, GMC Akola, India)
}

\begin{abstract}
:
Background :- Tuberculosis is a major public health problem in India and other developing countries. Extrapulmonary TB represents a greater diagnostic problem than pulmonary TB because it presents with less frequency and occurs with little liberation of bacilli, as well as the fact that it is localized in sites that are difficult to access.

Aims of study:- 1.To find out positivity of acid fast bacilli in aspiration smears of lymph nodes and other suspected tubercular lesions by Ziehl Neelson method and fluorescence method.

2. To evaluate the accuracy of fluorescent staining in the detection of Acid Fast Bacilli.

Study design:- In a Prospective study for the period of 2 years i.e. 2012 to 2014.

Method:-128 clinically suspected cases of tuberculous lesions attending the Department of Medicine, Surgery, ENT, Paediatrics, TB and Chest diseases were studied. Fine-needle aspiration was performed in these cases in the Department of Pathology at tertiary hospital, The results obtained after staining the smears using the Hematoxylin \& Eosin stain, Papanicolaou stain, Ziehl-Neelsen stain and Auramine-Rhodamine fluorescent stain were evaluated Data was analyzed by SPSS version 10.

Observation:- Out of 128 cases, 05 cases were diagnosed as reactive lymphadenopathy, 04 cases were acute suppurative inflammation and 04 samples were inadequate for evaluation. Statastical analysis of 115 samples was performed. A statistically significant difference was seen in the detection of acid fast bacilli by the AR stain, with a highly significant $p$ value. AR stain had detected additional 17/115 (14.7\%) cases which were ZN negative.

Conclusions: The fluorescent microscopy using Auramine-Rhodamine stain detects more AFB as compared to conventional ZN stain.
\end{abstract}

Keywords : AFB,ZN staining, Fluorescent microscopy and Mycobacterium Tuberculosis.

\section{Introduction}

According to TB-WHO India, India accounts for $1 / 5$ th of the global TB incident cases. Each year nearly 2 million people in India develop TB, of which around 0.87 million are infectious cases. Annual incidence in India is 1.96 million, out of which $20 \%$ constitute extrapulmonary tuberculosis. It is estimated that annually around 3,30,000 Indians die due to TB.1Extrapulmonary tuberculosis (EPTB) accounts for approximately $40 \%$ of tuberculosis cases. Extrapulmonary TB constitutes about 15-20\% of all cases of tuberculosis in immuno-competent patients and accounts for more than 50\% cases in HIV positive individuals. 2 Though not communicable, it is a significant cause of morbidity. Extrapulmonary TB represents a greater diagnostic problem than pulmonary TB because it presents with less frequency and occurs with little liberation of bacilli, as well as the fact that it is localized in sites that are difficult to access. 3 The key to control TB is its rapid detection. 4

Fine-Needle Aspiration Cytology Has Become A Popular Tool In Giving A Rapid Diagnosis Within A Matter Of 20 Minutes And Is Valuable As A Screening Procedure In Patients With Suspected Mycobacterial Infection, Particularly In Superficial, Easily Accessible Locations Such As Cervical And Submandibular Lymph Nodes. 5Varied Cytomorphologic Appearances Of Tuberculosis Have Been Reported On FNAC In Literature 6:-I.. Granulomatous Inflammation Consisting Of Epithelioid Granulomas With Or Without Langhan's Giant Cells, Ii. Smears Without Granulomas Showing Predominantly Necrotic Material Occasional Degenerated Epithelioid Cell With Or Without Neutrophil.

Diagnosis of tuberculous lymphadenitis still faces many challenges as the above appearances may be a morphological picture of other diseases, for example: necrosis can be a feature of the malignant neoplasm ${ }^{7}$, a 
full proof evidence of tuberculosis can only be made by the detection of acid-fast organism as the cause for granulomatous inflammation.In this study, two methods of detecting acid-fast organism is being used for optimal results to detected the presence of acid-fast bacilli (AFB) that are First is Ziehl-Neelsen (ZN) method and second byFluorescent method

Ziehl-Neelsen staining method is the most important tool in the diagnosis of tuberculosis in direct microscopic examination of appropriately stained smears for Acid-fast bacillus. 4 Even though this technique is simple, inexpensive and detects those cases of tuberculosis, which are infectious, the drawback of this method it that it is a time consuming process for staining as well as examination. False-negative results are possible, especially in paucibacillary cases. If more than 20 examinations are attempted, visual fatigue will lead to a deterioration of reading quality. Establishment of fluorescence microscopy is recommended by the World Health Organization (WHO) in such situations. 8 The most important advantage of fluorescence microscopy technique is that the slides can be examined at a lower magnification, thus allowing the examination of a much larger area per unit of time. In fluorescence microscopy, the same area that needs examination for 10 minutes with a light microscope, can be examined in two minutes. The tubercle bacilli stand out as bright objects against a dark background. Fluorescence microscopy, which makes them easily identifiable hence causing less eyestrain. The efficacy of fluorescence microscopy has proved to be much higher than conventional light microscopy and culture. ${ }^{8,9}$

\section{Material And methods}

A Prospective study was conducted at the Pathology Department of tertiary care hospital. Fine-needle aspiration was performed in these cases in the Department of Pathology at tertiary hospital, for the period of 2 years i.e. 2012 to 2014. 128 clinically suspected cases of tuberculous lesions attending the Department of Medicine, Surgery, ENT, Paediatrics, TB and Chest medicine were studied.

\subsection{Inclusion Criteria}

1. Patients with palpable swellings suspected to be tubercular in nature i.e. Patients having a history of swelling of more than 3-4 weeks duration and not responding to antibiotic treatment.

2. Purulent or necrotic material in the aspiration of the above mentioned swelling.

\subsection{Exclusion Criteria}

1. Frankly hemorrhagic aspirate.

2. Acellular smears/smears with crushed morphology or poorly stained slides will be excluded.

\subsection{Operational Definitions}

2.3.1 Granulomatous Inflammation:-It is defined on cytology as aggregates of epithelioid cells forming a granuloma with or without necrosis. Sometimes multinucleated giant cells are also seen. Based on the cytomorphology these were further classified as

Group- I - consisting of with epithelioid granulomas with or without Langhan's giant cells.

Group- II- showed predominatlly necrotic material, occasional degenerated epithelioid cells with or without neutrophils. ${ }^{6}$

\subsubsection{Positive for AFB by $\mathrm{ZN}$}

- $\quad$ On ZN staining the acid fast bacilli would be labeled when we find pink, beaded, and rod-shaped organisms after comparing with control samples.

\subsubsection{Positive for AFB by AR}

- On AR staining the acid fast bacilli would be labelled when we found a rod shaped reddishyellow fluorescence against dark background after comparing it with control samples.

2.4 Data Collection Procedure:-Patients fulfilling inclusion and exclusion criterion were selected from Fine needle aspiration cytology. After informed consent of patients and noting down the demographic data, fine needle aspiration (FNA) was performed in all suspected tubercular lesions. The method of FNA was same as described by Franzen, Koss and Frable. After explaining the FNA procedure to the patient written consent was taken. The patient used to lie in a supine position on the examination couch. The patient used to place in such a way that there was easy access to the swelling. Before performing FNA skin over the swelling was disinfected with spirit swabs. To perform FNAC 23 gauge needle with $20 \mathrm{ml}$ syringe was used. Consistency of the swelling was felt through the needle.4-5 smears were prepared on the glass slides. Slides prepared were fixed in $95 \%$ ethyl alcohol in Coplins Jar.Aspirate obtained was categorized as purulent and caseous. Purulent aspirate was yellowish, sticky material and caseous was whitish, granular material. Air dried slides were subjected for Z.N. 
and Fluorescent staining. Wet fixed smears were stained with H\&E and Papanicolaou stain.The findings of Hematoxylin and Eosin (H\&E) staining were categorized as Group I and Group II The finding of ZN staining was labeled as positive for AFB or negative for AFB. When the Z.N. stain does not detect AFB, then fluorescent stains, AR (Auramine-Rhodamine) was used for detection of AFB. Positive control- Smears of sputum positive for AFB by $\mathrm{ZN}$ method was stained with fluorescent dyes with each batch of sample slide. Data was analyzed by SPSS version 10.The present study was funded by Revised National Tuberculosis Control Programme (RNTCP)

\section{Observations}

128 clinically suspected cases of tuberculous lesions attending the Department of Medicine, Surgery, ENT, Paediatrics, TB and Chest diseases were studied. Fine-needle aspiration was performed in these cases in the Department of Pathology at tertiary hospital, for the period of 2 years i.e. 2012 to 2014. The results obtained after staining the smears using the Hematoxylin \& Eosin stain, Papanicolaou stain, Ziehl-Neelsen stain and Auramine-Rhodamine fluorescent stain were evaluated.Out of 128 cases, 05 cases were diagnosed as reactive lymphadenopathy, 04 cases were acute suppurative inflammation and 04 samples were inadequate for evaluation. Statical analysis of 115 samples was performed.

In the present study,table no 1 shows the age of the patients ranged from 2 months to 65 years. The maximum number of cases was in the age group of 21 to 30 years. There were $37(32.2 \%)$ out of 115 cases in the age group of 21-30 years. Extremes of age were least affected. There were $8(6.9 \%)$ out of 115 cases below 10 years and $5(4.3 \%)$ out of 115 cases above 50 years. Out of the 115 cases, 44 were male and 71 were female. A Female preponderance accounting for $71 / 115(61.7 \%)$ of cases, was seen. The male to female ratio was $1: 1.61$.

As per table no 2, The presenting complaint of patients Along with the swelling were, most of the patients presented with loss of appetite 56/115 (48.7\%). Only12/115 (10.4\%) patients presented with cough with expectoration.66/115 (57.4\%) cases gave the history of exposure to patients with tuberculosis. Out of $66,31 \mathrm{had}$ a family history and 35 cases suffered from tuberculosis in the past.Mantoux was performed in 34 cases. It was taken as positive when, there was more than $10 \mathrm{~mm}$ induration, after $48 \mathrm{hrs}$ with intradermal injection of PPD. It was positive in 31 cases $(91.2 \%)$ and negative in $3 / 34(8.8 \%)$ cases in which it was done.

The X-ray chest PA view was done in 35/115 (30.43\%) cases. 8/115 (6.9\%) cases showed lung parenchymal haziness. Only $1 / 115(0.9 \%)$ case showed cavitation. Maximum cases showed no significant radiological findings.

On local examination, swelling in maximum cases was soft to firm in consistency 89.6\% (103/115). Only $7 / 115(6.1 \%)$ cases were cystic. $4 / 115(3.4 \%)$ cases had sinus over the skin.Fine needle aspiration of suspected tubercular lesions was done. A lymph node was the most common site. Out of 115 cases 102 (88.7\%) cases were of lymphadenopathy. 6 cases of breast mass, 2 cases each from paraspinal and thyroid region and one case each from chest wall, 5th matacarpel and wrist. Out of 102 patients presented with lymphadenopathy. Cervical group of lymph nodes was most commonly affected. There were $83(81.4 \%)$ cases of cervical lymphadenopathy.

Yellowish sticky aspirate was labelled as purulent. Caseous aspirate was whitish and granular. In the present study, we obtained purulent aspirate in 62/115 (53.8\%) cases and in 53/115 (46.1\%) cases, aspirate were caseous.On Cytomorphological categorization of FNAC In the present study of the clinically suspected tuberculosis cases, the categorization of cytomorphological findings was done in 2 categories.

Group-I - With epithelioid granulomas. There were 51 (44.3\%) cases out of 115 cases in this group.

Group-II- Necrotic material or purulent material without granulomas, degenerated epithelioid cells and neutrophils. There were $64(55.7 \%)$ cases out of 115 cases in this group.

In the present study all the aspirated were categorized in two groups, purulent and caseous. On cytomorphology again we categorized in Group I and Group II. We found that more number of purulent aspirates were categorized in Group II 38/62 (61.2\%) as shown in table no 3.

Table 4 shows Results of ZN and AR staining were 42/115 (36.5\%) cases were positive with ZN. 59/115 $(51.3 \%)$ cases were positive with AR.Auramine-Rhodamine stain to detect more AFB as compared to ZN stain. A statistically significant difference was seen in the detection of acid fast bacilli by the AR stain, with a highly significant $p$ value. AR stain had detected additional 17/115 (14.7\%) cases which were ZN negative.

Table 4 shows Comparison of ZN and AR positivity in Group I and Group II were like Out of 115 cases, 51 cases were in group I. Out of these 51 cases, 11 were positive by $\mathrm{ZN}$ and 17 cases were positive by AR in group I. In the group II there were 64/115 cases, of which 31/64 and 42/64 were positive with ZN and AR respectively. But this difference was not of statistical significance. 


\subsection{Age \& Sex distribution:}

\section{Discussions}

As per table no 1, Most of the patients were in the age group of 21 to 30, accounting for 37 cases $(32.2 \%)$. The least affected age group was between 51 to 60 years, accounting for 5 cases (2\%). In a study by Thakur et al (2013), maximum cases affected were in the age group of 10-30 yrs, which accounted for $60 \%$ of total cases. ${ }^{10}$

In a study done by Kumar et al (1998), mean age of affected adults was 40.7 yrs. ${ }^{11}$.In their study of 209 cases, Ergete and Bekele observed a similar finding with the mean and median age of their patients being 22.8 and 23 years respectively. ${ }^{12}$ In our study mean age was 29.4 and median was 30 years. The age group most commonly affected by tuberculosis is comparable in all these studies performed at various places across the country. An individual is energetically most active during this period and therefore the chances of exposure are also more.

Ergete and Bekele also observed 87 males and 118 females with a male to female ratio of $1: 1.5{ }^{12}$ In the present study there were 44 male and 71 female. The male to female ratio in our study was $1: 1.6$.Female is more commonly affected with tuberculosis. This may be due to the well known fact that tuberculosis is more common in the malnourished people and in the developing country like India; females are more prone for malnourishment.

\subsection{Anatomical Location of FNA}

Of all the sites of aspiration, Lymph node was the most common site. There were 102 cases $(88.7 \%)$ of lymphadenopathy.

\subsection{Anatomical groups of lymph node involved:}

There were 83/115 (81.4\%) cases of cervical lymphadenopathy similar finding has also been observed by Annam et al (2009) ${ }^{13}$. In their study most of the lymph node aspirates were from cervical region, constituting $72 \%$ of the total aspirations performed. In the study done by Ergete and Bekele, there were 152/209 cases (72\%) of cervical lymphadenopathy. ${ }^{12}$ Thakur et al (2013) found that there was $83.3 \%$ cases of cervical lymphadenopathy. ${ }^{10}$

The present study also showed 62 purulent and 53 caseous aspirates.In the study on 70 patients, Shariff and Thomas (1991) ${ }^{7}$ observed the predominant pattern to be caseating tuberculosis in 40 cases. In their study they observed 11 aspirates as non-caseating tuberculosis and 17 of their cases showed acute necrotizing granulomatous inflammation. The presence of epithelioid cells and caseation are strong indicators of tuberculosis on morphology. Viable epithelioid cells were not seen in the group II of the present series and the confirmation of tuberculosis was based on the presence of bacilli in this group.

The present study also showed the AFB positivity was more in purulent than caseous aspirate. Present observations are in close agreement with those of Raghuveer ${ }^{14}$, Metre ${ }^{15} \&$ Bailey ${ }^{16}$ who have also found highest rate of AFB positivity of $66 \%, 80 \%$ and $87 \%$ respectively in necrotic material with or without granulomas. The reported accuracy of the FNA cytodiagnosis of tuberculosis affecting lymph nodes varies from $87.1 \%$ to $95.4 \%{ }^{17,18,19}$ in the literature reviewed.

\subsection{Comparison Of Ziehl-Neelsen \& Fluorescent Techniques:}

It is generally believed in literature that the Auramine-Rhodamine fluorescent technique yields more positive results than the conventional Ziehl-Neelsen method. This has been proved by the experiences of Traunt et al (1962) ${ }^{20}$, Needham et al (1957) ${ }^{21}$, Kuper et al (1960) ${ }^{22}$ and Braunstein et al (1961) ${ }^{23}$. Needham et al also stated that in his study group findings, $55 \%$ of cases which were negative on the conventional Ziehl-Neelsen method were proven to contain tubercle bacilli on the Auramine-Rhodamine fluorescent microscopy and confirmed by culture and/or animal inoculation. ${ }^{21}$ Previous studies on sputum smears have shown the positivity rates for both the Ziehl-Neelsen and Auramine-Rhodamine methods as $60 \%$ and $75 \%$ respectively. ${ }^{24}$ Annam et al (2009) ${ }^{13}$ proved on lymph node aspirates that the positivity rates were $44.11 \%$ and $81.37 \%$ by Ziehl-Neelsen and fluorescent techniques respectively.

In present study of 115 samples, the total AFB positivity rate was $36.5 \%(42 / 115)$ cases on $\mathrm{ZN}$ stain and was increased to $51.3 \%(59 / 115)$ on fluorescent stain. This comparison of Ziehl-Neelsen and fluorescent results showed a significant $\mathrm{p}$ value of $<0.05$ for the presence of bacilli. 
Figures and Tables

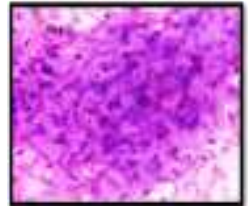

(a)

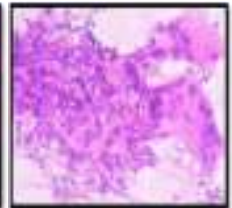

(b)

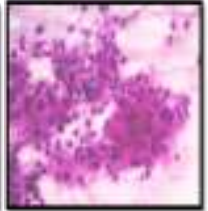

(c)

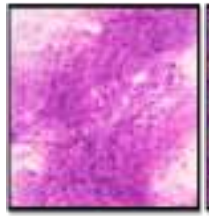

(d)

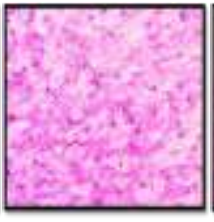

(e)

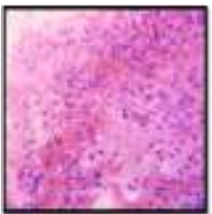

(f)

Group I

Group II

Fig 1:-Group I (a) Smear showing cluster of epithelioid cells and lymphocytes (H\&E stain x400) (b) Smear showing epithelioid cell cluster, giant cell and lymphocytes (H \& E stain x 400) (c) Clusters of epithelioid cells, lymphocytes and neutrophils (H \& E stain x 400)

Group II (d) Smear showing necrotic debris and lymphocytrs (H \& E stain x 400) (e) Smear showing necrotic background with lymphocytes and occasional degenerated epithelioid cell (H \& E stain x 400) (f) Smear showing planty of neutrophils and occasional degenerated epitheloid cell (H \& E stain x 400).

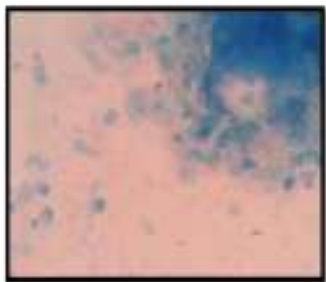

(a)

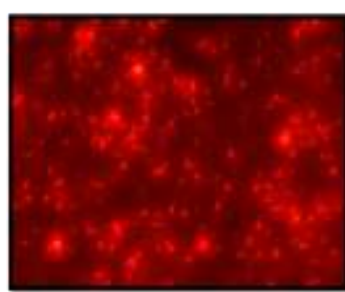

(b)

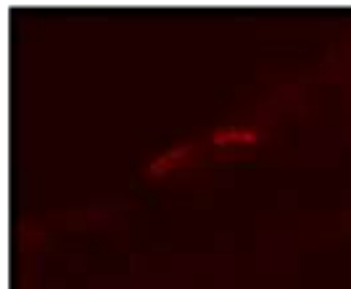

(c)

Fig 2 :- (a)FNA smear showing AFB (ZN stain x 1000);(b)FNA smear showing AFB in positive control (AR stain $x$ 400)White arrow showing artefact and blue arrow showing AFB;(c)FNA smears showing AFB (AR stain $\mathrm{x} 1500)$

Table 1: Age distribution

\begin{tabular}{|c|l|l|}
\hline Age in years & TOTAL & Percentage \\
\hline $0-10$ & 8 & 6.9 \\
\hline $11-20$ & 20 & 17.4 \\
\hline $21-30$ & 37 & 32.2 \\
\hline $31-40$ & 30 & 26.1 \\
\hline $41-50$ & 15 & 13.0 \\
\hline $51-60$ & 5 & 4.3 \\
\hline TOTAL & 115 & 100 \\
\hline
\end{tabular}

Mean Age: $29.4 \pm 12.59$ Median: 30 years Range (1-60 years)

Table 2: Presenting complaints

\begin{tabular}{|c|c|c|}
\hline $\begin{array}{c}\text { Presenting } \\
\text { complaints }\end{array}$ & Number & \% \\
\hline Cough with expectoration & 12 & 10.4 \\
\hline Low grade fever & 36 & 31.3 \\
\hline Loss of appetite & 56 & 48.7 \\
\hline Weight loss & 42 & 36.5 \\
\hline Swelling & 115 & 100 \\
\hline
\end{tabular}

Table 3: Correlation of Cytomorphology and type of aspirate

\begin{tabular}{|l|c|c|c|}
\hline $\begin{array}{l}\text { Cytomorphological } \\
\text { Groups }\end{array}$ & Purulent & Caseous & Total \\
\hline Group I & 24 & 27 & 51 \\
\hline Group II & 38 & 16 & 64 \\
\hline Total & 62 & 43 & 115 \\
\hline
\end{tabular}


Table 4: Results of ZN and AR staining

\begin{tabular}{|c|c|c|c|l|}
\hline \multirow{2}{*}{ Results } & \multicolumn{2}{|c|}{ ZN stain } & \multicolumn{2}{c|}{ AR stain } \\
\cline { 2 - 5 } & Number & Percent & Number & Percent \\
\hline Positive & 42 & 36.5 & 59 & 51.3 \\
\hline Negative & 73 & 63.5 & 56 & 48.7 \\
\hline TOTAL & 115 & 100 & 115 & 100 \\
\hline Mcnemar chi2=17.0 $\mathrm{p}<0.0001, \mathrm{HS}$ &
\end{tabular}

\section{Conclusion}

Fluorescent microscopy is rapid, simple, easy method for the detection of AFB. Detection rate for AFB of AR staining method is more than $\mathrm{ZN}$ method. Its superiority is ascribed to the ease with which bacilli are seen because of the greater contrast between bacilli and background With fluorescent microscopy larger number of smears can be screened in a short period of time. It is more useful especially when the sample or aspirates contain less number of bacilli (paucibacillary cases). Fluorochrome dyes are also easily available and not expensive. Staining procedure is very easy, but the problem is cost of equipment. Tuberculosis is so common in our country that we feel a wider use of the fluorescent microscopy will lead to greater detection rate than the conventiona

In laboratories where there is considerable load of work for detection of AFB, fluorescence microscopy is far better than the $\mathrm{ZN}$ technique; owing to its sensitivity and short period required to scan the whole smear.1 ZN staining method.

\section{References}

[1]. Global tuberculosis report 2013. World Health Organization.1-145.

[2]. Rosenblatt MB. Pulmonary tuberculosis: evolution of modern therapy. Bull N Y Acad Med 1973 Mar;49(3):163-96.

[3]. Garcia-Elorriaga G, Gracida-Osorno C, Carillo-Monster G, Gonzalez-Bonilla C. Clinical usefulness of the nested polymerase chain reaction in the diagnosis of extrapulmonary tuberculosis. Salud Publica Mex 2009; 51:240-245.

[4]. Narayana Reddy RA, Narayana SM, Shariff S. Role of fine-needle aspiration cytology and fluid cytology in extra-pulmonary tuberculosis. Diagn Cytopathol 2013 May;41(5):392-8.

[5]. Arora B, Arora DR. Fine needle aspiration cytology in diagnosis of tuberculous lymphadenitis. Indian J Med Res 1990 May;91:18992.

[6]. Kurekar K, Munshi M. Detection of acid fast bacilli in fine needle aspiration biopsies. Journal of cytology 1991-92;8(9):10-14.

[7]. Shariff S, Thomas JA. Fine needle aspiration cytodiagnosis of clinically suspected tuberculosis in tissue enlargements. Acta Cytol 1991 May-Jun;35(3):333-6.

[8]. Manual for sputum smear fluorescence microscopy. Central TB Division. Directorate General of Health Services. Ministry of Health and Family Welfare, Nirman Bhavan, Revised National Tuberculosis Control Programme.New Delhi.

[9]. Tarhan G, Ordulu L, Gümüşlü F, Ceyhan I, Cesur S. [Comparison of auramine-rhodamine and Erlich-Ziehl-Neelsen staining methods for the diagnosis of tuberculosis]. Mikrobiyol Bul 2003 Apr-Jun;37(2-3):131-6.

[10]. Thakur B, Mehrotra R, Nigam JS. Correlation of various techniques in diagnosis of tuberculous lymphadenitis on fine needle aspiration cytology. Patholog Res Int 2013.

[11]. Kumar N, Tiwari MC, Verma K. AFB staining in cytodiagnosis of tuberculosis without classical features: a comparison of ZiehlNeelsen and fluorescent methods. Cytopathology 1998 Jun;9(3):208-14

[12]. Ergete W, Bekele A. Acid fast bacilli in aspiration smear from tuberculous patients. Ethiop J Health Dev 2000; 14(1): 99-104

[13]. Annam V, Karigoudar MH, Yelikar BR. Improved microscopical detection of acid-fast bacilli by the modified bleach method in lymphnode aspirates. Indian J Pathol Microbiol 2009 Jul-Sep;52(3):349-52.

[14]. Rahuveer CV, Chetan M, Pai MR. Role of fine needle aspiration cytology in disorders of lymphnodes. J of Cytology 1996;13:45-9.

[15]. Metre MS, Jayaram G. Acid-fast bacilli in aspiration smears from tuberculous lymph nodes. An analysis of 255 cases. Acta Cytol 1987 Jan-Feb;31(1):17-9.

[16]. Bailey TM, Akhtar M, Ali MA. Fine needle aspiration biopsy in the diagnosis of tuberculosis. Acta Cytol 1985 Sep-Oct;29(5):7326.

[17]. Lau SK, Kwan S, Lee J, Wei WI. Source of tubercle bacilli in cervical lymph nodes: a prospective study. J Laryngol Otol 1991 Jul;105(7):558-61

[18]. Singh JP, Chaturvedi NK, Das A. Role of fine needle aspiration cytology in the diagnosis of tubercular lymphadenitis. Indian J Pathol Microbiol. 1989 Apr;32(2):100-4.

[19]. Nagpal BL, Dhar CN, Singh A, Bahl RA. Evaluation of imprint cytodiagnosis in cases of lymphadenopathy. Indian J Pathol Microbiol. 1982 Jan;25(1):35-9.

[20]. Truant JP, Brett WA, Thomas W Jr. Fluorescence microscopy of tubercle bacilli stained with auramine and rhodamine. Henry Ford Hosp Med Bull 1962Jun;10:287-96.

[21]. Needham GM. Comparative efficacy of direct microscopy (two methods) and cultures in the diagnosis of tuberculosis. Proc Staff Meet Mayo Clin 1957 Jan9;32(1):1-5.

[22]. Kuper SW, May JR. Detection of acid-fast organisms in tissue sections by fluorescence microscopy. J Pathol Bacteriol 1960 Jan;79:59-68.

[23]. Braunstein H and Adriano S M: Fluorescent stain for tubercle bacilli in histologic sections. Diagnostic efficiency in granulomatous lesions of lymph nodes. Am J Clin. Path 36:37, 1961.

[24]. Prasanthi K, Kumari AR. Efficacy of fluorochrome stain in the diagnosis of pulmonary tuberculosis co-infected with HIV. Indian J Med Microbiol 2005 Jul;23(3):179-81. 